\title{
Energy Efficiency of Laser Driven, Structure Based Accelerators
}

\author{
R. H. Siemann \\ Stanford Linear Accelerator Center, \\ Stanford University, Stanford, California, USA 94309
}

The acceleration efficiency of a laser driven linear accelerator is analyzed. The laser power, loss factor and impedances determine the maximum charge that can be accelerated and the efficiency of that acceleration. The accelerator structure can be incorporated into a laser cavity. The equation for the resultant laser pulse is derived and analyzed. A specific example is presented, and the steady-state laser pulse shapes, acceleration efficiency, and average unloaded gradient are calculated.

\section{Introduction}

Lasers have caught the imagination of scientists as possible accelerator power sources since their discovery. There is the potential of enormous accelerating gradients if the laser fields can be turned into longitudinal accelerating fields. This has led to research into a variety of possible acceleration mechanisms including non-linear laserparticle interactions, laser driven plasma waves, and laser driven vacuum acceleration. This manuscript is devoted to a specific manifestation of the latter - laser driven structures that are conceptually similar to RF driven linear accelerators.

Efficiency is as important an attribute as acceleration gradient for high energy physics because the luminosity of a high energy linear collider is proportional to beam power, and economical operation requires high efficiency. The acceleration efficiency of 
a laser driven structure depends on the properties of the structure itself. This is covered in the next section.

The efficiency can be increased significantly by incorporating the accelerator into the laser cavity. Energy that would otherwise be lost is recovered by doing this. Beam generated fields affect the laser cavity, and they must be included in the analysis. The intra-cavity accelerator is a major topic of this paper and is analyzed in section III.

\section{Passage of a Single-Bunch}

\section{Impedances and Loss Factor}

The first considerations are for the passage of a single, relativistic, point-like bunch through an infinitesimal length of accelerator structure. The situation is illustrated in Figure 1. The unloaded gradient, $G_{0}$, is related to the power flow by the characteristic impedance of the accelerating mode that depends on the accelerator geometry

$$
Z_{C}=\frac{G_{0}^{2} \lambda^{2}}{P} .
$$

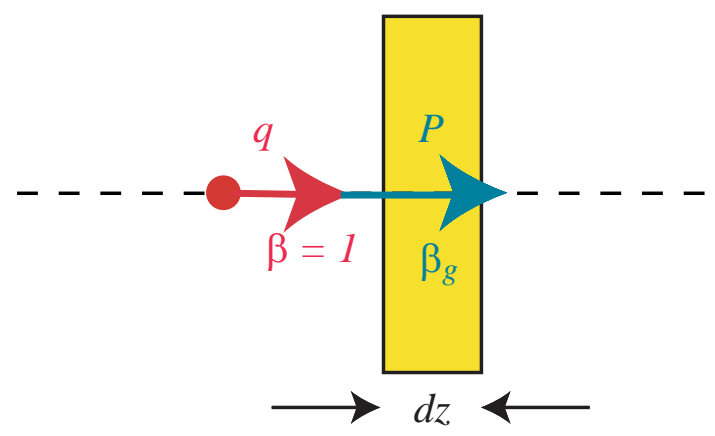

Figure 1: A relativistic, point-like bunch with charge $q$ passing through at infinitesimal length $d z$ of accelerator. $P$ is power in the accelerating mode, which has group velocity $\beta_{g}$ and phase velocity $\beta_{\text {phase }}=1$. 
The charge will radiate energy into the accelerating mode with a loss factor per unit length ${ }^{1}$

$$
k=\frac{1}{4} \frac{c \beta_{g}}{1-\beta_{g}} \frac{Z_{C}}{\lambda^{2}} .
$$

This produces a decelerating gradient $G_{F}=k q$ and fields in the narrow-band accelerating mode that interfere with the fields from the external power source.

In addition to these narrow-band fields, there will be radiation into a wide frequency band from processes such as Cherenkov radiation. As an example, an effective impedance for this radiation has been found by calculating the retarding field due to Cherenkov radiation in an infinite dielectric rod with a hole of radius $R$ in the center. ${ }^{2}$ The effective retarding gradient is

$$
G_{H}=\frac{q c Z_{H}}{\lambda^{2}}
$$

where

$$
Z_{H}=Z_{0} \frac{1}{2 \pi(R / \lambda)^{2}} .
$$

$Z_{H}$ is defined in this way to show the dependence on the dimensionless parameter $R / \lambda$.

This result holds for other geometries with $R$ being a characteristic separation from the particle to the structure boundary and with a factor of order unity multiplying the expression for $Z_{H} \cdot{ }^{3}$

\section{Optimum Efficiency and Accelerated Charge}

The beam-loaded gradient is

$$
G=G_{0}-G_{F}-G_{H}=\frac{\sqrt{P Z_{C}}}{\lambda}-q\left(\frac{c Z_{H}}{\lambda^{2}}+k\right),
$$

and the increase in the kinetic energy of the beam per unit length is 


$$
\frac{d U_{k i n}}{d z}=q G .
$$

The acceleration efficiency is the ratio of this increase to the electromagnetic energy per unit length $P / \beta_{g} c$ with a leading factor that accounts for the difference between the group and particle velocities. It is

$$
\eta=\frac{1}{\left(1-\beta_{g}\right)} \frac{\beta_{g} c}{P} \frac{d U_{k i n}}{d z} .
$$

The efficiency is an optimum when

$$
q=q_{\text {opt }}=\frac{G_{0}}{2\left(c Z_{H} / \lambda^{2}+k\right)},
$$

and for that charge the efficiency is

$$
\eta_{\max }=\frac{Z_{C} \beta_{g}}{4 Z_{H}\left(1-\beta_{g}\right)+Z_{C} \beta_{g}} .
$$

The efficiency and gradient for different charges are

$$
\eta=\eta_{\max } \frac{q}{q_{o p t}}\left(2-\frac{q}{q_{o p t}}\right)
$$

and

$$
G=\frac{\sqrt{P Z_{C}}}{\lambda}\left(1-\frac{q}{2 q_{o p t}}\right) .
$$

As an example, the photonic band gap fiber accelerator of X. E. $\operatorname{Lin}^{4}$ has $R=$ $0.678 \lambda, \lambda=1.05 \mu \mathrm{m}, \beta_{g}=0.60, Z_{C}=195 \Omega,\left(k=2.0 \times 10^{21} \mathrm{~V} / \mathrm{C}-\mathrm{m}\right)$, and $Z_{H} \sim 130 \Omega$ $\left(c Z_{H} / \lambda^{2}=3.5 \times 10^{22} \mathrm{~V} / C-m\right)$ when the crystal is approximated as a solid dielectric and variation of the dielectric constant with frequency is ignored. The damage threshold for a $10 \mathrm{~cm}$ long fiber was estimated to be $P=7.4 \mathrm{~kW}$, and the optimum charge and the efficiency at that power are $q_{\text {opt }} \sim 5 f C$ and $\eta_{\max } \sim 0.05$. This example shows that the maximum efficiency can be rather high, but the accelerated charge is low. The latter is a consequence of 1) the proportionality $q_{o p t} \propto \lambda$ in eq. (8) and 2) the structure having a 


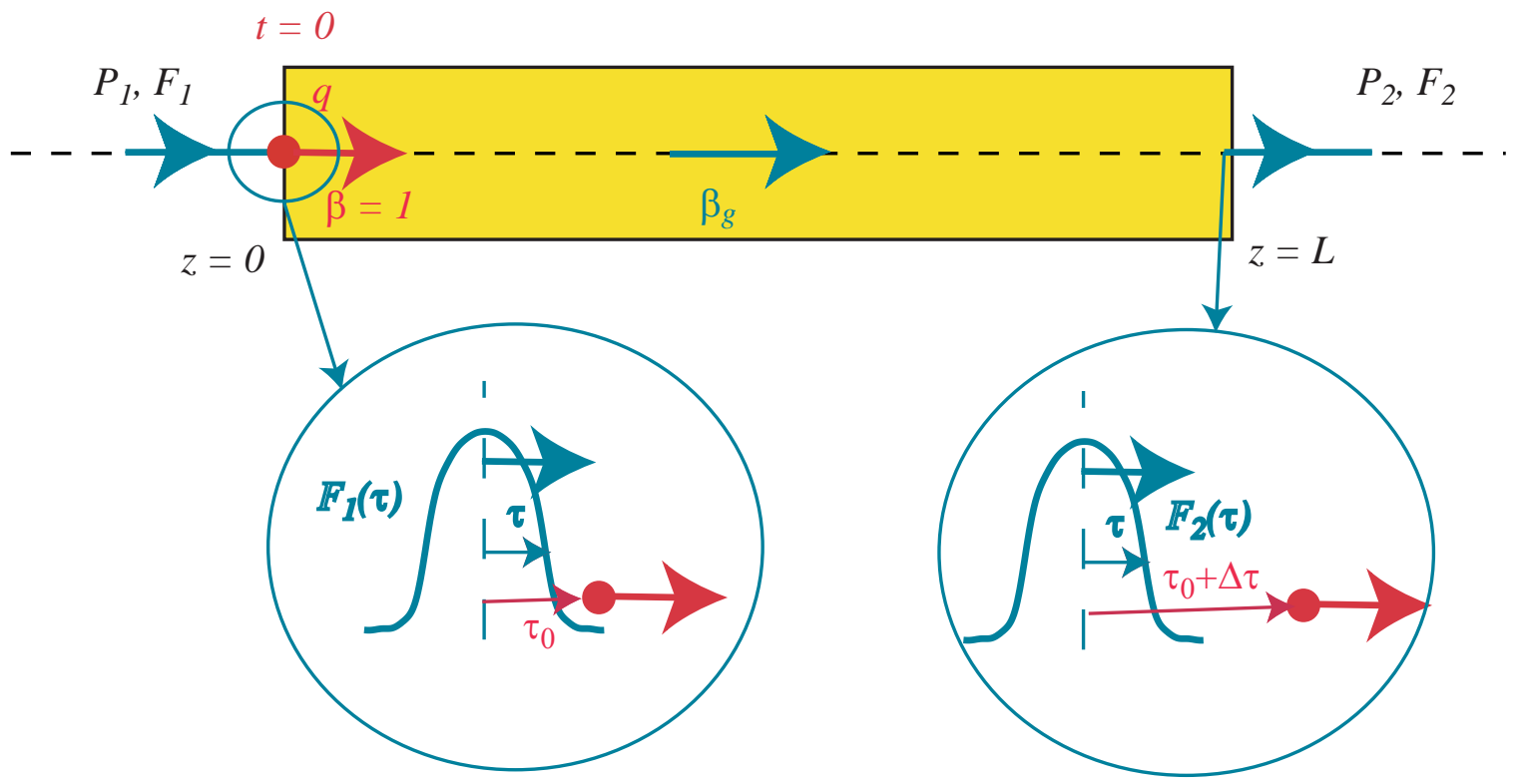

Figure 2: A beam bunch and accelerator structure of length $L . P_{1}$ and $P_{2}$ are the input and output power in the accelerating mode and $F_{1}$ and $F_{2}$ are the corresponding fields. The time coordinate $\tau$ is shown with $\tau>0$ to the right of the center line. The electron bunch is $\tau_{0}$ ahead of the center of the laser pulse at $z=0$ and $\tau_{0}+\Delta \tau$ ahead at $z=L$. The group velocity of $\beta_{g}$ is assumed to be constant for the length of the structure.

beam hole comparable to the wavelength resulting in large $Z_{C}$ and $Z_{H}$. One way to accelerate more charge in a single bunch would be to increase the characteristic size of the structure, thereby reducing $Z_{C}$ and $Z_{H}$, and using higher laser power to obtain the unloaded gradient. Some initial work in this direction has been reported by B. Cowan et $a l^{5}$

\section{Finite Length Structure}

The results above can be generalized to a structure of length $L$ and time-dependent input power, $P_{1}$, and field $F_{1}=\sqrt{P_{1} Z_{C}} / \lambda$ (Figure 2). The laser envelopes are functions of $\tau$, 
which is measured from a reference point on the pulse. The sign convention is the one that would follow from an instantaneous snapshot: $\tau>0$ is ahead of the reference point. The laser pulse and particle bunch propagate at different velocities, and, if the bunch is at $\tau_{0}$ at $z=0$, then at $z=L$ it is at

$$
\tau=\tau_{0}+\frac{L}{c} \frac{1-\beta_{g}}{\beta_{g}}=\tau_{0}+\Delta \tau .
$$

The average, effective unloaded gradient is

$$
\left\langle G_{0}\right\rangle=\frac{1}{\Delta \tau} \int_{\tau_{0}}^{\tau_{0}+\Delta \tau} F_{1}(\tau) d \tau .
$$

The change in the beam kinetic energy in passing through the structure is

$$
\Delta U_{k i n}=q L\left(\left\langle G_{0}\right\rangle-q\left(\frac{c Z_{H}}{\lambda^{2}}+k\right)\right),
$$

and the effective unloaded gradient should be used in the expression for the optimum charge

$$
q_{\text {opt }}=\frac{\left\langle G_{0}\right\rangle}{2\left(c Z_{H} / \lambda^{2}+k\right)} .
$$

The beam produces fields in the accelerating mode. One manifestation of this is the "self-field" that decelerates the source charge with gradient $G_{F}$. Another is fields radiated out of the downstream end of the accelerator given by

$$
E_{b}(\tau)=-2 k q\left(\Theta\left(\tau-\tau_{0}\right)-\Theta\left(\tau-\left(\tau_{0}+\Delta \tau\right)\right)\right) \equiv-2 k q S\left(\tau, \tau_{0}\right) .
$$

where $\Theta$ is the step function. The fields produced near the downstream end of the accelerator arrive the earliest when the bunch arrives at $\tau=\tau_{0}+\Delta \tau$, and the fields produced at the beginning propagate at the group velocity and arrive at $\tau=\tau_{0}$. This expression is shown to satisfy energy conservation in Appendix A. 


\section{Intra-Cavity, Actively Mode Locked Accelerator}

\section{Accelerator/Laser Cavity Equation}

The intra-cavity, actively mode-locked laser driven accelerator is illustrated in Figure 3. The accelerator structure is incorporated into a laser cavity consisting of a gain medium, AM modulator, and four mirrors. Three of the four mirrors are assumed to be completely reflecting. The fourth mirror, the one located at position 3 , is a partially reflecting beam combiner, and an external laser beam is injected into the cavity at that mirror. Losses, other than those associated with the beam combiner, are included and parameterized by a loss factor $\delta$. The laser envelope at location $n$ is $F_{n}(\tau)$ where $\tau$ is measured from the center of the pulse

The particle beam is a train of bunches of charge $q$ spaced at the round-trip laser period, $T$, which includes the dispersive effects of the gain medium. Bunches are $\tau_{0}$ ahead of the corresponding laser pulse at location 1, and phases are defined by taking the phase of the beam current as zero.

This cavity is an actively mode-locked laser with seeding in the accelerating mode from the beam induced field and, in addition, from the external laser. An equation for $F_{1}(\tau)$ is developed in Appendix B by closely following A. Siegman's treatment of active mode-locked homogenous lasers. ${ }^{6}$ It is

$$
\begin{aligned}
\frac{d^{2} F_{1}(\tau)}{d \tau^{2}} & -\frac{\mathrm{M} \omega_{m}^{2}\left(\delta \omega_{a}\right)^{2} \tau^{2}}{8 \alpha_{m} p_{m}} F_{1}(\tau)+\frac{\left(1+\alpha_{m} p_{m}-\delta-\lambda / r\right)\left(\delta \omega_{a}\right)^{2}}{4 \alpha_{m} p_{m}} F_{1}(\tau) \\
& =\frac{\left(\delta \omega_{a}\right)^{2}}{4 r \alpha_{m} p_{m}}\left(2 k q r S\left(\tau, \tau_{0}\right)-\sqrt{1-r^{2}} E_{\text {ext }}(\tau) e^{i\left(\phi_{e x t}+\pi / 2\right)}\right)
\end{aligned}
$$

where $\lambda$ is the eigenvalue for the steady state solution. 


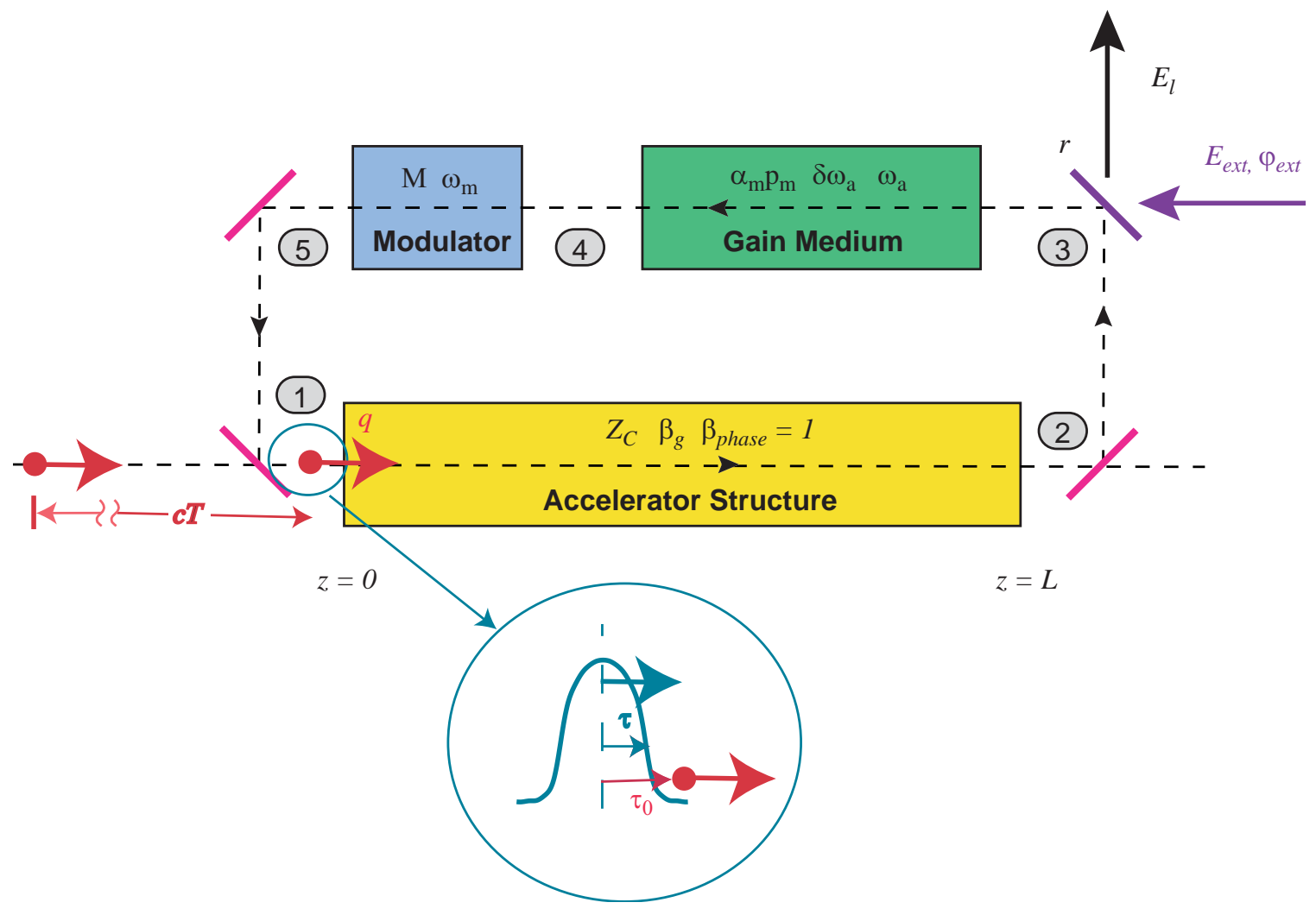

Figure 3: The intra-cavity, actively mode-locked laser driven accelerator. The parameters of the different elements are discussed in the text, and the subscripts $1, \ldots, 5$ refer to the locations indicated in the cavity.

The solution of the equation starts with the eigenmodes of the homogenous equation. They are orthonormal Hermite functions

$$
h_{n}(u)=\frac{1}{\sqrt{2^{n} n ! \pi^{1 / 2}}} \exp \left(-\frac{u^{2}}{2}\right) H_{n}(u)
$$

with $u=\tau / \sigma_{\tau} \cdot{ }^{7}$ The $H_{n}(u)$ are Hermite polynomials, and for any $n$

$$
\sigma_{\tau}=\sqrt[4]{\frac{8 \alpha_{m} p_{m}}{\mathrm{M} \omega_{m}^{2}\left(\delta \omega_{a}\right)^{2}}}
$$

The eigenvalues are

$$
\lambda_{n}=r\left(1+\alpha_{m} p_{m}-\delta-\frac{4(2 n+1) \alpha_{m} p_{m}}{\left(\delta \omega_{a} \sigma_{\tau}\right)^{2}}\right)
$$


This result has been obtained by D. M. Kim et al. ${ }^{8}$ The mode that lases, the one with the largest eigenvalue, is a Gaussian shaped pulse $F_{1}(u) \sim h_{0}(u)$.

The solution of the full equation (17) is a sum of Hermite functions. ${ }^{9}$ Multiplying by $\sigma_{\tau}^{2}$ and expressing the fields as a function of $u$, the equation becomes

$$
\begin{gathered}
\frac{d^{2} F_{1}(u)}{d u^{2}}-u^{2} F_{1}(u)+\frac{\left(1+\alpha_{m} p_{m}-\delta-\lambda / r\right)\left(\delta \omega_{a} \sigma_{\tau}\right)^{2}}{4 \alpha_{m} p_{m}} F_{1}(u) \\
=\frac{\left(\delta \omega_{a} \sigma_{\tau}\right)^{2}}{4 r \alpha_{m} p_{m}}\left(2 k q r S\left(u, u_{0}\right)-\sqrt{1-r^{2}} E_{e x t}(u) e^{i\left(\phi_{e x t}+\pi / 2\right)}\right)
\end{gathered}
$$

The solution can be written in a number of ways that have different limits as the right hand side of eq. (21) goes to zero. ${ }^{9}$ The solution that approaches a Gaussian pulse with peak accelerating field $G_{p k}$ is

$$
F_{1}(u)=\pi^{1 / 4} G_{p k} \sum_{n=0}^{\infty} \frac{\gamma_{n} h_{n}(u)}{\gamma_{0}-2 n} .
$$

The expansion coefficients $(n=0,1, \ldots)$ are given by

$$
\begin{aligned}
\gamma_{n} & =\frac{\left(\delta \omega_{a} \sigma_{\tau}\right)^{2}}{4 r \alpha_{m} p_{m} \pi^{1 / 4} G_{p k}} \int_{-\infty}^{\infty}\left(2 k q r S\left(u, u_{0}\right)-\sqrt{1-r^{2}} E_{e x t}(u) e^{i\left(\phi_{e x t}+\pi / 2\right)}\right) h_{n}(u) d u \\
& =\frac{\left(\delta \omega_{a} \sigma_{\tau}\right)^{2}}{4 r \alpha_{m} p_{m}}\left(\frac{2 k q r}{\pi^{1 / 4} G_{p k}} \int_{u_{0}}^{u_{0}+\Delta \tau / \sigma_{\tau}} h_{n}(u) d u-\frac{\sqrt{1-r^{2}}}{\pi^{1 / 4} G_{p k}} e^{i\left(\phi_{e x t}+\pi / 2\right)} \int_{-\infty}^{\infty} E_{e x t}(u) h_{n}(u) d u\right)^{,}
\end{aligned}
$$

and the relation between $\gamma_{0}$ and $\lambda$ is

$$
\lambda=\lambda_{0}-\frac{4 r \alpha_{m} p_{m} \gamma_{0}}{\left(\delta \omega_{a} \sigma_{\tau}\right)^{2}} .
$$

\section{No External Laser}

First consider the case where $r=1$. The circulating fields are completely reflected at the beam combiner, and the external laser beam does not couple into the cavity. If $\phi_{f}$ is the phase of the laser field with respect to the beam current, the eigenvalue is 

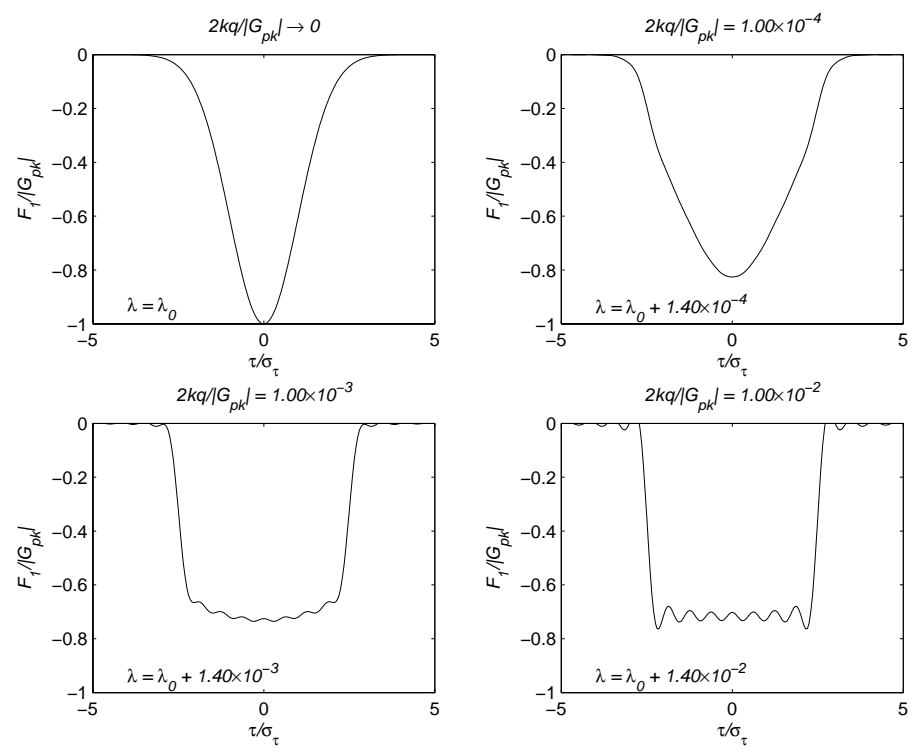

Figure 4: Laser pulse shapes for $r=1$ and different beam charges. This calculation was performed with $\left(\delta \omega_{a} \sigma_{\tau}\right)^{2} / \alpha_{m} p_{m}=1 \times 10^{4}, \tau_{0}=-2.5 \sigma_{\tau}$, and $\Delta \tau=5 \sigma_{\tau}$. The sum in eq. (22) was truncated at $n=50$.

$$
\lambda=\lambda_{0}-\frac{2 k q e^{-i \phi_{f}}}{\pi^{1 / 4}\left|G_{p k}\right|} \int_{u_{0}}^{u_{0}+\Delta \tau / \sigma_{\tau}} h_{0}(u) d u .
$$

The integral is positive independent of the location of the beam with respect to the envelope of the laser pulse, and the eigenvalue is a maximum when the laser field is $\pi$ out of phase with the beam current, i.e. at the phase for maximum deceleration. The beam is decelerated rather than accelerated!

The equilibrium pulse shape can be calculated using the equations above. The quantity $\left(\delta \omega_{a} \sigma_{\tau}\right)^{2} / 4 \alpha_{m} p_{m}$ is proportional to $\delta \omega_{a} / \omega_{m}$ and is much greater than one, and, therefore, the wakefield determines the pulse shape even for modest beam charge. Results are shown in Figure 4. For example, the case when $2 k q / G_{p k}=0.001$ would correspond to $q \sim 0.04 q_{\text {opt }}$ in Lin's structure. ${ }^{4}$ 


\section{Solution with the External Laser}

These results, in particular that the laser lases with the beam at the decelerating phase, are not surprising since this is the phase of the field seeding the laser. The external laser is necessary to change this and bring the beam to the accelerating phase.

The calculation that follows uses a particular external laser pulse shape for definiteness, $E_{e x t}=\pi^{1 / 4} E_{p k} h_{0}(u)$, although the expressions in eqs. (22) - (24) are general. The eigenvalue, assuming that the laser lases in phase with the beam current $\left(\phi_{f}=0\right)$, is

$$
\lambda=\lambda_{0}+\left(\frac{E_{p k} \sqrt{1-r^{2}}}{G_{p k}} e^{i\left(\phi_{e x t}+\pi / 2\right)}-\frac{2 k q r}{\pi^{1 / 4} G_{p k}} \int_{u_{0}}^{u_{0}+\Delta \tau / \sigma_{\tau}} h_{0}(u) d u\right) .
$$

The assumption about the phase is correct provided the quantity in parentheses, which is proportional to the integral of the entire pulse seeding the laser cavity, is positive. This requires $\phi_{\text {ext }}=-\pi / 2$. The cavity will lase in phase with the beam current in the region below and to the right of the dashed line in Figure 5.

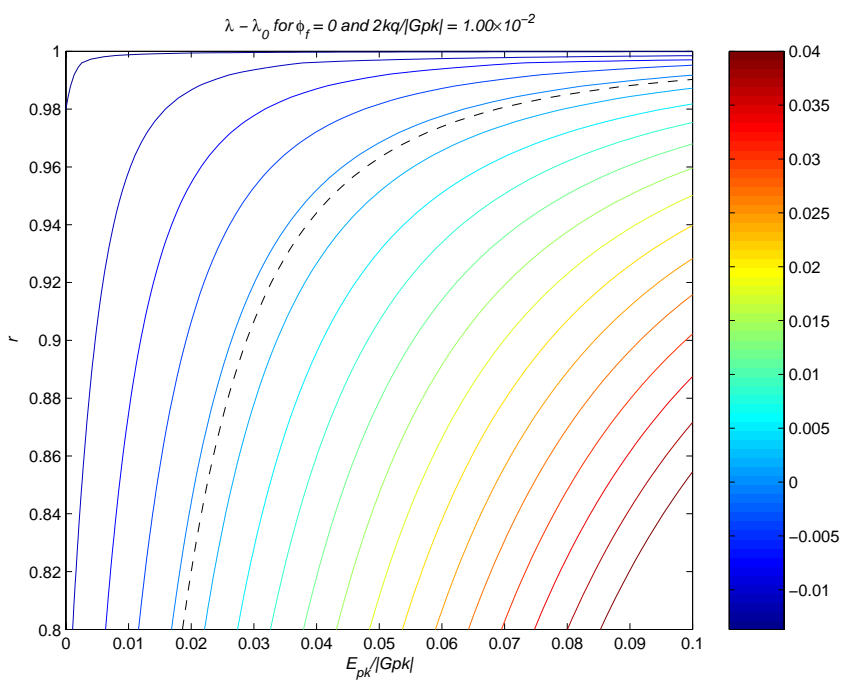

Figure 5: Eigenvalue for $2 k q / G_{p k} \mid=0.01,\left(\delta \omega_{a} \sigma_{\tau}\right)^{2} / \alpha_{m} p_{m}=1 \times 10^{4}, \tau_{0}=-2.5 \sigma_{\tau}$, and $\Delta \tau=5 \sigma_{\tau}$ assuming the beam is at the accelerating phase,$\phi_{f}=0$. The dashed black line is $\lambda=\lambda_{0}$. 
The expansion coefficients are

$$
\gamma_{n}=\frac{\left(\delta \omega_{a} \sigma_{\tau}\right)^{2}}{4 \alpha_{m} p_{m}}\left(\frac{2 k q}{\pi^{1 / 4} G_{p k}} \int_{u_{0}}^{u_{0}+\Delta \tau / \sigma_{\tau}} h_{n}(u) d u-\delta_{n, o} \frac{E_{p k}}{G_{p k}} \frac{\sqrt{1-r^{2}}}{r}\right),
$$

and the average unloaded gradient can be expressed in terms of them

$$
<G_{0}>=\pi^{1 / 4} G_{p k} \sum_{n=0}^{\infty} \frac{\gamma_{n}}{\gamma_{0}-2 n} \frac{\sigma_{\tau}}{\Delta \tau} \int_{u_{0}}^{u_{0}+\Delta \tau / \sigma_{\tau}} h_{n}(u) d u
$$

Placing the accelerator in the laser cavity does not affect the maximum accelerated charge because that limit arose from considerations of the decelerating gradient. The benefit of having it there is an increase the acceleration efficiency by reducing the input energy needed to reach the same unloaded gradient. The kinetic energy gain of the beam is given by eq. (14), and the acceleration efficiency is

$$
\eta=\frac{\Delta U_{k i n}}{\frac{\lambda^{2} \sigma_{\tau}}{Z_{C}} \int_{-\infty}^{\infty} F_{1}^{2}(u) d u}\left(\frac{\int_{-\infty}^{\infty} F_{1}^{2}(u) d u}{\sqrt{\pi} E_{p k}^{2}+\int_{-\infty}^{\infty}\left(F_{1}^{2}(u)-F_{3}^{2}(u)\right) d u}\right) .
$$

The single passage efficiency, $\eta_{0}$, is multiplied by a factor, $\Xi_{\eta}$, giving the efficiency increase. The first term in the denominator of $\Xi_{\eta}$ corresponds to energy from the external laser, and the second term is the energy added by the gain medium.

\section{A Specific Example}

A specific example is chosen for illustrating the application of these results. The gain medium is Nd:YAG with $\delta \omega_{a}=2 \pi \times 120 \mathrm{GHz}$. The voltage gain coefficient and modulation are chosen to give $\sigma_{\tau}=40 \mathrm{psec}$ and $\left(\delta \omega_{a} \sigma_{\tau}\right)^{2} / \alpha_{m} p_{m}=1 \times 10^{4}$. The accelerator structure is that of X. E. $\mathrm{Lin}^{4}$ described above. For this example, the starting position of the bunch is $\tau_{0}=-2.5 \sigma_{\tau}$, and interaction time is $\Delta \tau=5 \sigma_{\tau}$. These give an accelerator length $L=7.5 \sigma_{\tau} c=9 \mathrm{~cm}$, and the average unloaded gradient without recycling is $G_{p k} / 2$. 
The peak accelerating gradient is limited by material damage. The damage fluence, $\Phi_{\text {damage, }}$, and peak gradient for a Gaussian pulse, $G_{p k, n}$, are related by

$$
G_{p k, n r}=\frac{G_{p k}}{E_{\max }} \sqrt{\frac{\Phi_{\text {damage }} Z_{0}}{\sqrt{\pi \varepsilon_{r}} \sigma_{\tau}}} .
$$

The quantity $\varepsilon_{r}$ is the dielectric constant, and the ratio $G_{p k} / E_{\max }$ is the ratio of the peak gradient to the maximum electric field in the dielectric $(=1 / 2.1$ for Lin's structure). Using this and the results of B. C. Stuart et al for the damage fluence in fused silica ${ }^{10}$ $\left(\Phi_{\text {damage }}=12.5 \mathrm{~J} / \mathrm{cm}^{2}\right.$ for $\left.\sigma_{\tau}=40 \mathrm{psec}\right)$ the peak accelerating gradient is $G_{p k, n r}=320$ $\mathrm{MeV} / \mathrm{m}$. The average unloaded gradient is $\left\langle G_{0}\right\rangle=160 \mathrm{MeV} / \mathrm{m}$, and the optimum charge without recycling using eq. (15) is $q_{\text {opt }}=2.2 \mathrm{fC}$.

The damage fluence limit is the same with the accelerator in the laser cavity. That gives a relation between $G_{p k}$ and $\gamma_{0}$

$$
G_{p k, n r}^{2}=G_{p k}^{2} \sum_{n=0}^{\infty}\left(\frac{\gamma_{n}}{\gamma_{0}-2 n}\right)^{2}
$$

The average gradient depends on $G_{p k}$ and increases as $G_{p k} \rightarrow G_{p k, n r}$. $E_{p k}$ and $r$ can be determined by maximizing the efficiency once $G_{p k}$ and $\gamma_{0}$ are determined. Figure 6 shows the results of this calculation for one value of charge, $q \sim q_{\text {opt }}$. The efficiency approaches $\eta \sim 0.5$ at high gradient where approximately half of the input energy accelerates the beam and the other half is radiated away as Cherenkov radiation.

Pulse shapes are shown in Figure 7 for different values of $\left\langle G_{0}\right\rangle$. The pulse broadens and there are regions of decelerating gradient that shrink in length and magnitude as the average unloaded gradient increases.

The efficiency can be calculated versus bunch charge and loss parameter $\delta$. The results are shown in Figure 8. When $\delta=0$ the only losses in the cavity are due to the 
beam combiner and energy radiated away as Cherenkov radiation, and the efficiency decreases linearly with charge as the Cherenkov radiation increases. When $\delta \neq 0$ there are additional losses associated with energy circulating in the cavity, and they are the dominant loss mechanism at low charge making $\eta \rightarrow 0$ as $q \rightarrow 0$. However, even with $\delta$ $=0.05$ there is substantial gain in efficiency for $q \sim q_{\text {opt }}$.

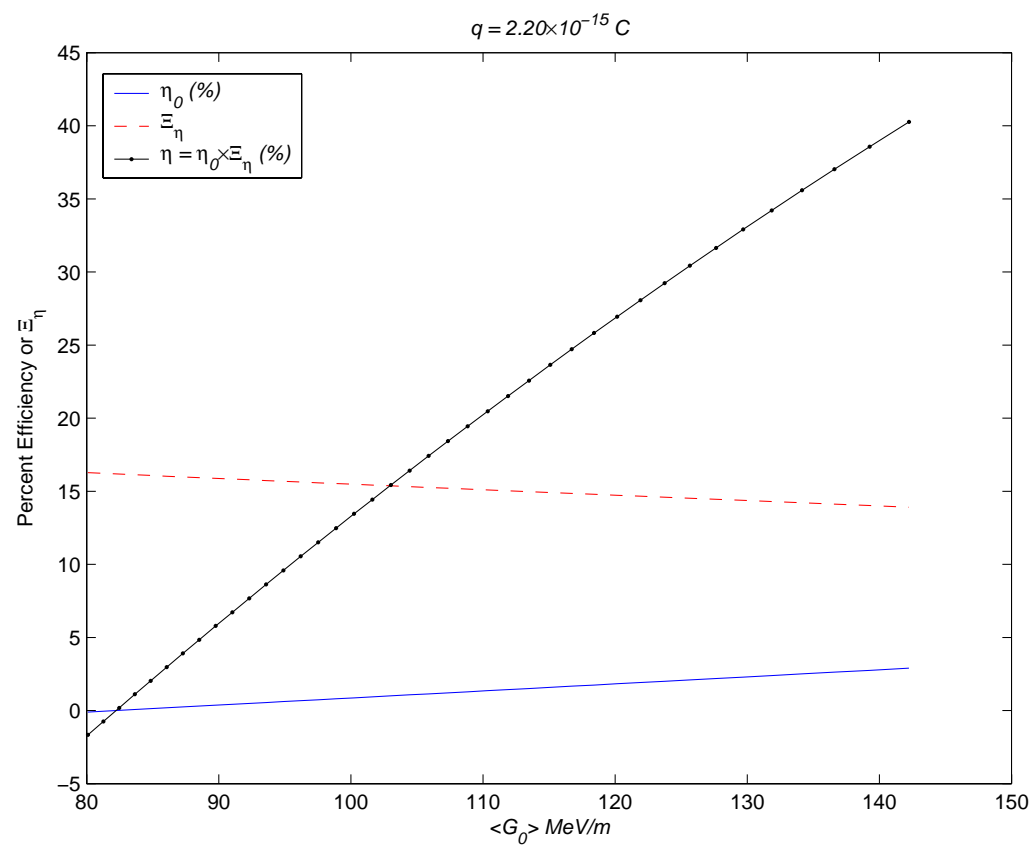

Figure 6: Efficiency without and with energy recycling for $q=2.2 \mathrm{fC}$ and $\delta=0$. The efficiency has been maximized for each value of $\left\langle G_{0}>\right.$ by trading-off $r$ and $E_{p k}$. For example, at $<G_{0}>=140 \mathrm{MeV} / \mathrm{m} r=0.912$ and $E_{p k} / G_{p k}=0.439$. Below $<G_{0}>\sim 82 \mathrm{MeV}$ the retarding gradient is greater than the unload gradient, and, therefore, $\eta<0$. 


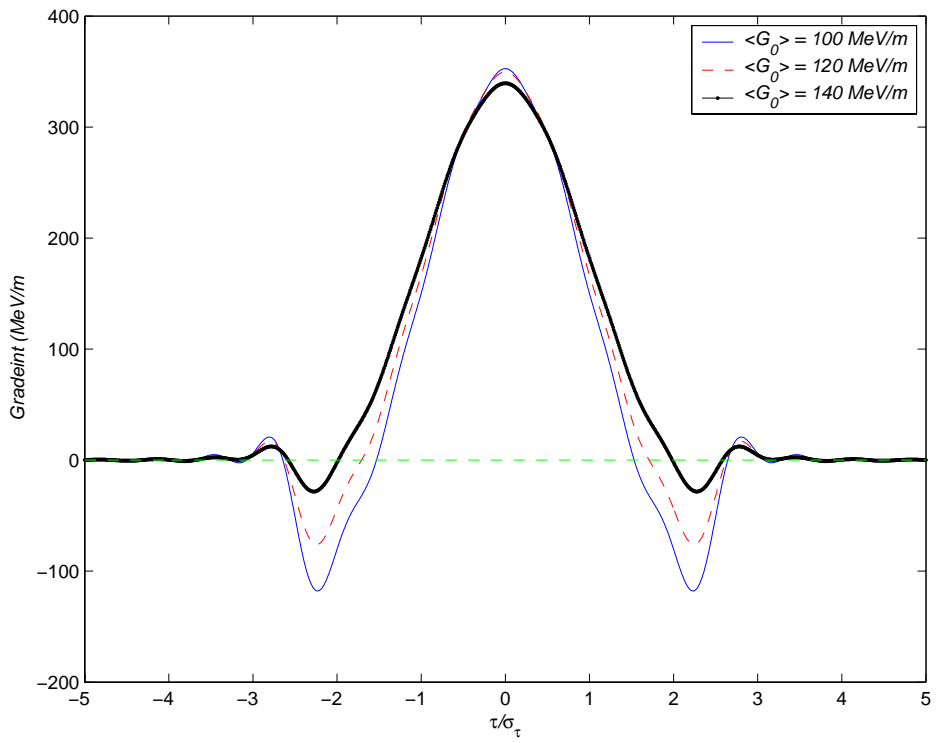

Figure 7: Pulse shapes for different values of $\left\langle\boldsymbol{G}_{0}\right\rangle$.

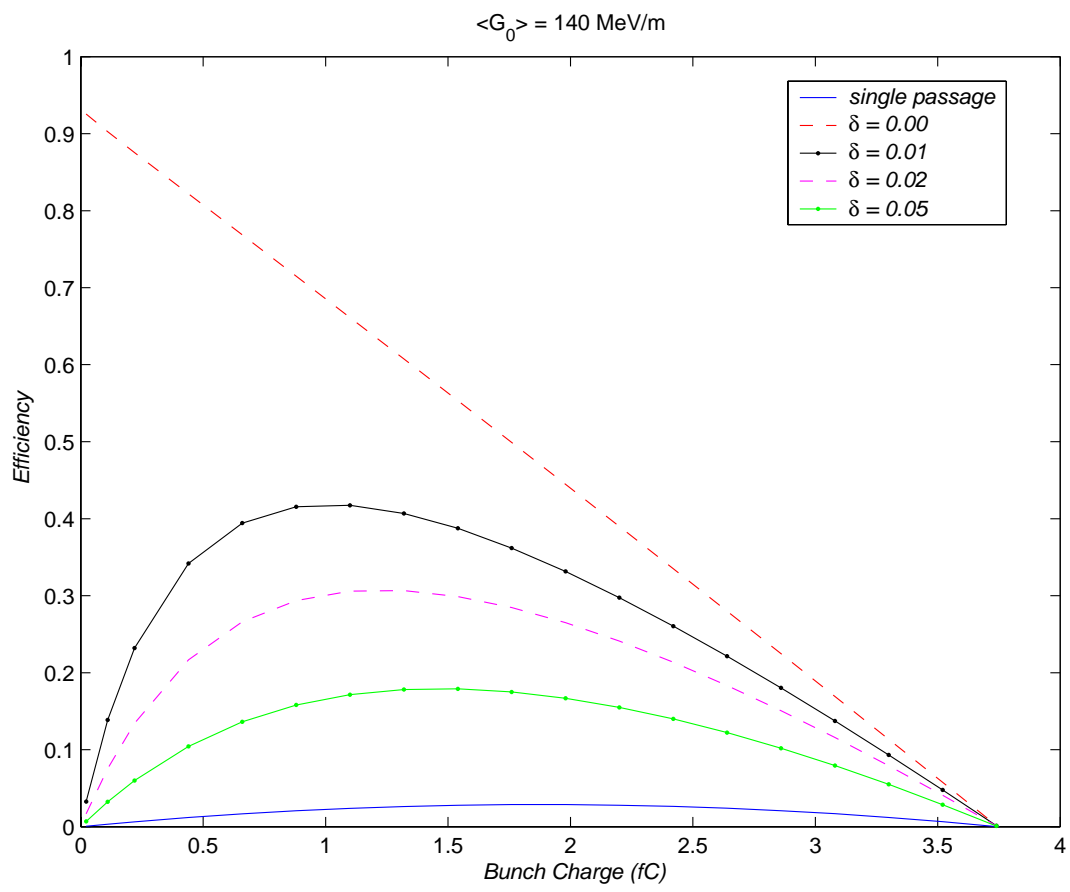

Figure 8: Efficiency as a function of bunch charge for $\left\langle G_{0}>=140 \mathrm{MeV} / \mathrm{m}\right.$ and different values of the loss parameter.

Page 15 of 21 


\section{Summary and Conclusion}

We have analyzed the efficiency of a laser driven, structure based accelerator. It has been shown that laser power and impedances determine 1) the maximum single pass efficiency and 2) the optimum and maximum single bunch charges that can be accelerated. Those charges are small because the impedances depend on wavelength as $\lambda^{-2}$ although it should be possible to increase them with structures that have low impedances and still reach high gradients by using large laser powers.

However, high repetition rates and multiple beam bunches per laser pulse are likely to be necessary to reach beam powers of interest for a high energy linear collider. The former is natural for lasers, and the latter can be analyzed using the methods in this paper.

High acceleration efficiency is another requirement for large beam powers, and it has been shown that incorporating the accelerator into the laser cavity can significantly improve the efficiency. The practicality of that will depend on the losses in the input and output couplers.

Future research will follow the directions indicated above.

The early phases of this work benefited from collaboration with L. Schächter. Conversations with E. Colby, B. Cowan, and T. Plettner have been very helpful to my understanding of laser/particle beam system.

This work was supported in part by U.S. Department of Energy Grant No. DE-AC0376SF00515. 


\section{Appendix A: Energy Conservation and Beam Produced Fields}

Consider the situation illustrated in Figure 2. The input power with an arbitrary timedependence produces an unloaded gradient

$$
F_{1}(\tau)=\frac{1}{\lambda} \sqrt{Z_{C} P_{1}(\tau)}
$$

at the upstream end of the structure. The output pulse shape would be the same if there were no beam and no losses in the structure.

The beam itself produces field in the accelerating mode. At the exit of the structure this field is given by

$$
E_{b}(\tau)=-q E_{w}\left(\Theta\left(\tau-\tau_{0}\right)-\Theta\left(\tau-\left(\tau_{0}+\Delta \tau\right)\right)\right) .
$$

This expression includes the facts that the field is proportional to the beam charge $q$, extends from $\tau=\tau_{0}$ to $\tau=\tau_{0}+\Delta \tau$, and is decelerating. This appendix contains a derivation leading to the proportionality constant $E_{w}$.

The output power is given by

$$
P_{2}(\tau)=\frac{\lambda^{2}}{Z_{C}}\left(F_{1}(\tau)+E_{b}(\tau)\right)^{2} .
$$

Energy conservation applied to the beam passage is

$$
U_{\text {in }}+\int_{-\infty}^{\infty} P_{1}(\tau) d \tau=U_{\text {out }}+q G_{H} L+\int_{-\infty}^{\infty} P_{2}(\tau) d \tau
$$

where $U_{\text {in }}$ and $U_{\text {out }}$ are the initial and final kinetic energies of the bunch and the second term on the right-hand-side is the energy in the wide-band radiation. Using eqs. (3) and (14) results in

$$
q L\left(\left\langle G_{0}\right\rangle-G_{F}\right)=\int_{-\infty}^{\infty} P_{i n}(\tau) d \tau-\int_{-\infty}^{\infty} P_{\text {out }}(\tau) d \tau .
$$

Substituting eq. (34) for $P_{\text {out }}$ and using the expression for $G_{F}$ and eq. (33) 


$$
q L\left(\left\langle G_{0}\right\rangle-k q\right)=-\frac{\lambda^{2} q^{2} E_{w}^{2}}{Z_{C}}\left(\frac{L\left(1-\beta_{g}\right)}{\beta_{g} c}\right)+\frac{\lambda q E_{w}}{\sqrt{Z_{C}}} \int_{\tau_{0}}^{\tau_{0}+\Delta \tau} \sqrt{P_{\text {out }}(\tau)} d \tau .
$$

Equating either the terms proportional to $q$ or the terms proportional to $q^{2}$ gives

$$
E_{w}=2 k
$$

as would be expected from P. Wilson's "Fundamental Theorem of Beam Loading". 11

\section{Appendix B: Equation for the Intra-Cavity, Actively Mode Locked}

\section{Accelerator}

The procedure is to follow a laser pulse around the cavity shown in Figure 3. Quantities are expressed in the time domain as functions of $\tau$ or in the frequency domain as functions of the angular frequency, $\omega$, and the subscript $n$ denotes locations in Figure

3. Zero phase is defined by the beam current.

The calculation starts at location 1 with $\tilde{F}_{1}(\omega)$. The beam produces a field, given by eq. (16), in the accelerator structure that interferes $\tilde{F}_{1}$ at the accelerator exit

$$
\tilde{F}_{2}(\omega)=\tilde{F}_{1}(\omega)-2 k q \tilde{S}\left(\omega, \tau_{0}\right)
$$

where $\tilde{S}\left(\omega, \tau_{0}\right)$ is the Fourier transform of $S\left(\tau, \tau_{0}\right)$ with respect to $\tau$, and the minus sign accounts for the phase of the beam generated fields.

The beam combiner is described by a unitary $4 \times 4$ matrix that relates the incoming and outgoing electric fields in Figure $9^{12}$

$$
\left(\begin{array}{l}
b_{1} \\
b_{2} \\
b_{3} \\
b_{4}
\end{array}\right)=\left(\begin{array}{cccc}
0 & r & i \sqrt{1-r^{2}} & 0 \\
r & 0 & 0 & i \sqrt{1-r^{2}} \\
i \sqrt{1-r^{2}} & 0 & 0 & r \\
0 & i \sqrt{1-r^{2}} & r & 0
\end{array}\right)\left(\begin{array}{l}
a_{1} \\
a_{2} \\
a_{3} \\
a_{4}
\end{array}\right)
$$

where $\mathrm{r}$ is the reflection $a_{2} \rightarrow b_{1}$. Therefore, the field at location 3 is 


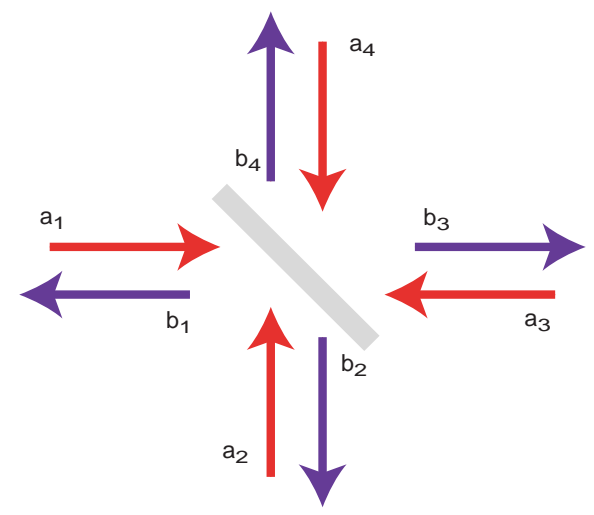

Figure 9: The a's and b's are the complex amplitudes of the incoming and outgoing electric fields, respectively.

$$
\tilde{F}_{3}(\omega)=r \tilde{F}_{2}(\omega)+\sqrt{1-r^{2}} \tilde{E}_{e x t}(\omega) e^{i\left(\phi_{e x t}+\pi / 2\right)}
$$

where $\phi_{\text {ext }}$ is the phase of the external laser field with respect to the beam. The electric field lost from the cavity at the beam combiner is

$$
\tilde{E}_{l}(\omega)=i \sqrt{1-r^{2}} \tilde{F}_{2}(\omega)+r \tilde{E}_{e x t}(\omega) e^{i \phi_{\text {ext }}}
$$

The laser gain medium has a complex voltage gain

$$
\tilde{g}(\omega) \approx 1+\alpha_{m} p_{m}-\delta-\frac{4 \alpha_{m} p_{m} \omega^{2}}{\left(\delta \omega_{a}\right)^{2}} .
$$

In this equation $\delta \omega_{a}$ is the linewidth and $\alpha_{m} p_{m}$ is the voltage gain coefficient that, in the steady state, equals the total cavity loss. For convenience the losses due to imperfections are included in this equation as a factor $e^{-\delta} \approx 1-\delta$. The field at the exit of the gain medium is

$$
\tilde{F}_{4}(\omega) \approx \tilde{F}_{3}(\omega)\left(1+\alpha_{m} p_{m}-\delta-\frac{4 \alpha_{m} p_{m} \omega^{2}}{\left(\delta \omega_{a}\right)^{2}}\right) .
$$

The modulator is taken to be an AM modulator with modulation depth $M$ and angular frequency $\omega_{m}$. Its effect is to create sidebands at $\pm \omega_{m}$ 


$$
\tilde{F}_{5}(\omega)=(1-\mathrm{M}) \tilde{F}_{4}(\omega)+\frac{\mathrm{M}}{2}\left(\tilde{F}_{4}\left(\omega-\omega_{m}\right)+\tilde{F}_{4}\left(\omega+\omega_{m}\right)\right) \approx \tilde{F}_{4}(\omega)+\frac{\mathrm{M} \omega_{m}^{2}}{2} \frac{d^{2} \tilde{F}_{4}}{d \omega^{2}} .
$$

Combining these equations and keeping the lowest order terms gives

$$
\begin{gathered}
\tilde{F}_{5}(\omega)=r\left(1+\alpha_{m} p_{m}-\delta\right) \tilde{F}_{1}(\omega)+\frac{r \mathrm{M} \omega_{m}^{2}}{2} \frac{d^{2} \tilde{F}_{1}(\omega)}{d \omega^{2}} \\
-\frac{4 r \alpha_{m} p_{m} \omega^{2}}{\left(\delta \omega_{a}\right)^{2}} \tilde{F}_{1}(\omega)+\left(\sqrt{1-r^{2}} \tilde{E}_{e x t}(\omega) e^{i\left(\phi_{e x t}+\pi / 2\right)}-2 k q r \tilde{S}\left(\omega, \tau_{0}\right)\right)
\end{gathered} .
$$

Finally, transforming to the time domain and looking for steady-state solutions $F_{5}(\tau)=\lambda F_{1}(\tau)$ gives the eigenvalue equation

$$
\begin{aligned}
\frac{d^{2} F_{1}(\tau)}{d \tau^{2}} & -\frac{\mathrm{M} \omega_{m}^{2}\left(\delta \omega_{a}\right)^{2} \tau^{2}}{8 \alpha_{m} p_{m}} F_{1}(\tau)+\frac{\left(1+\alpha_{m} p_{m}-\delta-\lambda / r\right)\left(\delta \omega_{a}\right)^{2}}{4 \alpha_{m} p_{m}} F_{1}(\tau) \\
& =\frac{\left(\delta \omega_{a}\right)^{2}}{4 r \alpha_{m} p_{m}}\left(2 k q r S\left(\tau, \tau_{0}\right)-\sqrt{1-r^{2}} E_{e x t}(\tau) e^{i\left(\phi_{e x t}+\pi / 2\right)}\right)
\end{aligned}
$$

1 K. L. F. Bane and G. Stupakov, Phys. Rev. ST Accel. Beams 6, 024401 (2003).

2 G. Dôme, Second European Accelerator Conference, Nice, France, 628, (1990), edited by P. Marin \& P. Mandrillon, Editions Frontieres, Les Ulis, France (1990).

${ }^{3}$ L. Schächter, R. L. Byer, and R. H. Siemann, Tenth Advanced Accelerator Concepts Workshop, Mandalay Beach, CA, 310, (2002), edited by C. Clayton \& P. Muggli, AIP, Melville, NY (2002).

4 Xintian Eddie Lin, Phys. Rev. ST Accel. Beams 4, 051301 (2001).

5 B. Cowan, M. Javanmard, and R. Siemann, 2003 Particle Accelerator Conference, Portland, OR, 1855, (2003), edited by J. Chew, P. Lucas and S. Webber, IEEE, Piscataway, NJ, (2004).

${ }^{6}$ Anthony E. Siegman, Lasers (University Science Books, Sausalito, CA, 1986), Chap. 27, p.1061 ff. 
7 Philip M. Morse and Herman Feshbach, Methods of Theoretical Physics (McGraw-Hill Book Co, New York, 1953), Chap. 8, p.904.

${ }^{8}$ Dae M. Kim, S. Marathe, and T. A. Rabson, J. Appl. Phys. 44, 1673 (1973).

9 Philip M. Morse and Herman Feshbach, Methods of Theoretical Physics (McGraw-Hill Book Co, New York, 1953), Chap. 12, p.1647.

${ }^{10}$ B. C. Stuart, M. D. Feit, A. M. Rubenchik, B. W. Shore, and M. D. Perry, Phys. Rev. Lett. 74, 2248 (1995).

11 P. Wilson, Physics of High Energy Particle Accelerators, Batavia, IL, 450, (1981), edited by R. A. Carrigan, F. R. Huson \& M. Month, , AIP, Melville, NY (1982).

12 Anthony E. Siegman, Lasers (University Science Books, Sausalito, CA, 1986), Chap. 11, p.398 ff. 